\title{
Las superficies artificiales en la Raya Central Ibérica \\ El uso de modelos lineales generalizados para definir los factores condicionantes de los cambios territoriales
}

\author{
Francisco Javier JARAíz CABANILLAS ${ }^{1}$ \\ jfjaraiz@unex.es \\ Julián MORA AlisedA ${ }^{2}$ \\ jmora@unex.es \\ José Antonio GutiÉRREZ GALLEGo ${ }^{3}$ \\ jagutier@unex.es
}

Recibido: 2 de febrero del 2012

Enviado a evaluar: 6 de febrero del 2012

Aceptado: 27 de julio del 2012

\section{RESUMEN}

Los cambios que viene experimentando la Raya Central Ibérica y que son impulsados por la concentración de la población en las principales ciudades, el abandono de los espacios rurales, el desarrollo de nuevas infraestructuras de transporte, el dinamismo unido a los nuevos cultivos de regadío, etc., motivan cambios en las superficies artificales.

Se desarrolla un análisis espacial aplicado a determinar las principales fuerzas causantes de los cambios en las superficies artificiales en una región transfronteriza como la citada. Los cambios de cobertura y uso del suelo fueron cuantificados con técnicas estadísticas e imágenes del proyecto CORINE Land Cover (CLC) y los factores condicionantes se analizaron modelos lineales generalizados $(\mathrm{glm})$ combinando variables humanas y geofísicas.

Las conclusiones muestran la idoneidad del empleo de los datos del proyecto CLC, la significatividad de las variables independientes escogidas, y la constatación de la validez de los glm para la explicación de los cambios de cobertura del suelo de un determinado territorio.

Palabras clave: Raya Central Ibérica, superficies artificiales, cambios de cobertura y uso del suelo, CORINE, modelos lineales generalizados.

1 Investigador de la Universidad de Extremadura. Departamento de Arte y Ciencias del Territorio. Facultad de Filosofía y Letras. Av. de la Universidad s/n, 10071 Cáceres.

2 Profesor Titular de Universidad (UEX). Departamento de Arte y Ciencias del Territorio.

3 Profesor Titular de Universidad (UEX). Departamento de Expresión Gráfica.. 


\title{
Artificial surfaces in the Raya Central Ibérica Use generalized linear models to define driving forces of the territorial changes
}

\begin{abstract}
The rapid changes that the socio-demographic structure and the economic activity are experiencing in the "Raya Central Ibérica", and that are produced for the new socioeconomic needs, the concentration of the population in the principal cities, the abandon of the rural spaces, the growth of irrigation areas, etc., they motivate changes in the artificial surfaces.

A spatial analysis is applied to determine the principal driving forces of the artificial surfaces changes. For this, different tools are in use in order to differentiate the land use and land cover changes or the driving forces. The changes were quantified by statistical technologies and images of CORINE Land Cover Project and the driving factors were analyzed by logistic regressions (generalized linear models) combining human and geophysics variables.

Conclusions show the suitability of the employment of the information of CORINE Land Cover project, the significant selected independent variables, and the validity of the generalized linear models for the explanation of the land use and land cover change of a certain territory.
\end{abstract}

Keywords: Raya Central Ibérica, artificial surfaces, land use and land cover changes, CORINE, generalized linear models.

\section{Les artificielles surfaces dans la Raya Central Ibérica L'usage de modèles linéaires généralisés pour définir les facteurs condicionantes des changements territoriaux}

\section{RÉSUMÉ}

Les changements que la Raya Central Ibérica expérimente et que sont poussés par la concentration de la population dans les villes principales, l'abandon des espaces ruraux, le développement de nouvelles infrastructures de transport, le dynamisme joint aux nouvelles cultures d'irrigation, etc., motivent des changements sur les surfaces artificales.

Une analyse spatiale se développe appliqué à déterminer les forces causantes des changements sur les artificielles surfaces dans une région transfrontalière comme la citée. Les changements de couverture et l'usage du sol ont été quantifiés par des techniques statistiques et des images du projet CORINE Land Cover (CLC) et des facteurs condicionantes les modèles linéaires répandus ( $\mathrm{glm}$ ) ont été analysés en combinant variables humaines et géophysiques.

Les conclusions montrent l'aptitude de l'emploi des données du projet CLC, l'importance des indépendantes variables choisies, et la constatation de la validité des glm pour l'explication des changements de couverture du sol d'un territoire déterminé.

Mots clé: Raya Central Ibérica, les changements de couverture et l'usage du sol, CORINE, des modèles linéaires répandus. 


\section{INTRODUCCIÓN}

La finalidad última del estudio es comprender el impacto de las actividades económicas y del medio físico sobre el territorio, más concretamente sobre los espacios ocupados por las nuevas superficies artificiales y los efectos del cambio socio-demográfico y territorial motivado por esto, prestando una especial atención a las nuevas estructuras espaciales, la evolución de los usos del suelo y a las repercusiones implícitas en las transformaciones que se hayan producido.

Para ello, el estudio se centrará fundamentalmente en tratar de caracterizar los cambios de coberturas y usos del suelo en general, en explicar las modificaciones en el territorio debido al desarrollo de nuevas superficies artificiales y definir los posibles efectos de los cambios.

Se utiliza para ello técnicas de análisis de datos como los modelos lineales generalizados y bases de datos númericos y geográficos elaboradas a partir de imágenes de satélite y facilitadas por el Proyecto CORINE Land Cover (CLC en adelante). Las fuentes de información serán las estadísticas producidas diferentes instituciones oficiales, mientras que para el estudio que se pretende llevar a cabo se tendrá como unidad administrativa de análisis el municipio en el caso español y el concelho en el caso portugués. Puede considerarse que el empleo del término municipal como unidad de análisis limita los resultados y las conclusiones, no obstante, el estudio de la expansión urbana en cada uno de los núcleos que pueden constituir un municipio no es el objetivo del estudio.

No puede ser obviado que este análisis forma parte de un estudio más amplio y global, en el que no sólo se tratan los cambios de usos artificiales, sino además los cambios de usos agrarios y los cambios de coberturas forestales y de agua. La selección pues, en este artículo de las superficies artificiales, como variable dependiente o respuesta, responde a la intención de exponer la situación de estos usos en el área de estudio. Un área transfronteriza eminentemente rural, en la que, pese a la débil estructura urbana y a la muy baja densidad demográfica la superficie artificial creció a nivel regional y porcentualmente, de un modo considerable. No obstante, cuando se analiza el crecimiento de las superficies artificiales a nivel municipal, y se compara la expansión de la marcha artificial con el total de la superficie de los términos, se aprecia la verdadera dimensión del fenómeno.

Por consiguiente, la aportación novedosa de este trabajo de investigación, no es exclusivamente el estudio de las superficies artificiales o los diferentes usos de estas como tal, sino el seguimiento, la explicación y las repercusiones de los cambios de usos artificiales que se están produciendo. En esta línea surge la idea de hacer un seguimiento de la evolución de estos usos del suelo y su explicación. Idea que se plasma en el Programa CORINE Land Cover (de hay su empleo), destinado a crear una herramienta para la toma de decisiones en materia de gestión del medio ambiente y los recursos naturales.

Como objetivos concretos del análisis de los cambios en las superficies artificiales y los factores condicionantes de estos cambios en el área de estudio, se deben subrayar:

1. Obtener información detallada y actualizada de los cambios de cobertura y uso del suelo en el área el estudio, concretamente las superficies artificiales, su distribución, su evolución y sus diferentes detonantes. 
2. Crear, gestionar, analizar e interpretar una amplia base de datos territoriales, socioeconómicos y ambientales.

3. Optimizar el análisis e interpretación de los registros históricos.

4. Generar cartografía temática de resultados.

Son muy importantes también los objetivos metodológicos de la investigación:

1. Utilizar e implementar técnicas como los modelos lineales generalizados, para la definición de los factores condicionantes de los cambios de usos artificiales, mediante el empleo de variables independientes socioeconómcias y físicas.

2. Emplear los Sistemas de Información Geográfica (SIG) para la generación de una cartografía temática fácilmente interpretable.

\section{2. ÁREA DE ESTUDIO}

A pesar de la indefinición del concepto de frontera y su alcance espacial, sobre los que no hay unanimidad ni unicidad en el uso que de los mismos se hace, se ha optado por estudiar los cambios de cobertura de las superficies artificiales en el territorio que comprende a las dos provincias de Extremadura (Cáceres y Badajoz) y a los cinco distritos portugueses fronterizos con la región española (Guarda, Castelo Branco, Portalegre, Évora y Beja). Este espacio transfronterizo se ha denominado por diversos autores como Raya Central Ibérica (Cabero et al., 1995-1996; Campesino, 1997, 2007; López et al., 1997; Mora, 1997) y, por lo tanto, ésta es la definición que se emplea en el artículo. No obstante hay que subraya que los límites de este espacio oscilan de unos autores a otros, aunque la esencia territorial perdura en todo ellos.

Como ya se ha señalado con anterioridad, esta investigación se encuadra dentro de una general en la que no sólo se tratan las superficies artificiales, sino además, los usos agrícolas y las coberturas forestales y de agua. El objeto por el cual se selecciona un espacio transfronterizo para el estudio como es la Raya Central Ibérica, responde a la necesidad de conocer si el comportamiento de las diferentes coberturas y usos del suelo siguen un patrón común en un área regida por la misma política comunitaria en materia agraria e infraestructural y caracterizada por similares particularidades físicas: geomorfología, geología, edafología, clima, etc. El territorio no comprende de divisiones administrativas arbitrarias muchas veces establecidas sin criterios coherentes. Así pues, se trata de ver el comportamiento a ambos lados de la Raya de las coberturas y usos del suelo, concretamente en este caso, las superficies artificales.

En la Raya Central Ibérica, espacio perteneciente a tres regiones Objetivo 1 de la UE, los fondos estructurales y de cohesión que se aplican para corregir los desequilibrios territoriales han alterado considerablemente este espacio. Esta zona que se analiza es objeto de una política comunitaria que la viene casi configurando desde la óptica socioeconómica a través de iniciativas como INTERREG, y está posibilitando la creación de infraestructuras y equipamientos de todo tipo, que influyen en su funcionalidad y permeabilidad, y en la alteración de las diferentes coberturas del suelo.

Los distritos y provincias y los municipios o concelhos que configuran este espacio pueden apreciarse en la Figura 1. De igual modo, en este mapa se distinguen las 
manchas de los núcleos de población con mayor entidad en cuanto al número de habitantes. Entre estas ciudades podría destacarse Badajoz (148.334 hab.), Cáceres (93.131 hab.) y Mérida (56.395 hab.), en territorio extremeño; o Évora (54.780 hab.), Castelo Branco (53.909 hab.) y Covilhã (52.101 hab.) en los distritos portugueses.

Puede sorprender la elección tanto de los municipios y concelhos como de los distritos y las provincias para unidades administrativas para el análisis, pero todo ello responder a criterios bien definidos. En el primer caso, decir que no se seleccionaron las freguesías como unidad base para Portugal (quizá más acorde en cuanto al tamaño de los municipios) porque las variables independientes o explicativas que se pretendían utilizar, tan sólo estaban disponibles a nivel municipal y de concelho en los Institutos Nacionales de Estadística de ambos países. En cuanto a la elección de los distritos y las provincias la solución final que primó fue una relacionada con la expresión gráfica de la cartografía. Pese a que los distritos es una división administrativa en desuso en Portugal, su elección en lugar de una división más acorde con la realidad administrativa europea como pueden ser la NUTS III, y que se corresponderías con las subregiones portuguesas y las provincias extremeñas, responde a un aspecto de límites territoriales. Las NUTS III portuguesas son mucho menos extensas que las extremeñas. Por consiguiente, como los límites de los distritos y las provincias eras similares, se decidió analizar las dos provincias extremeñas y los cinco distritos portugueses fronterizos.

Figura 1. Municipios y/o concelhos del área de estudio

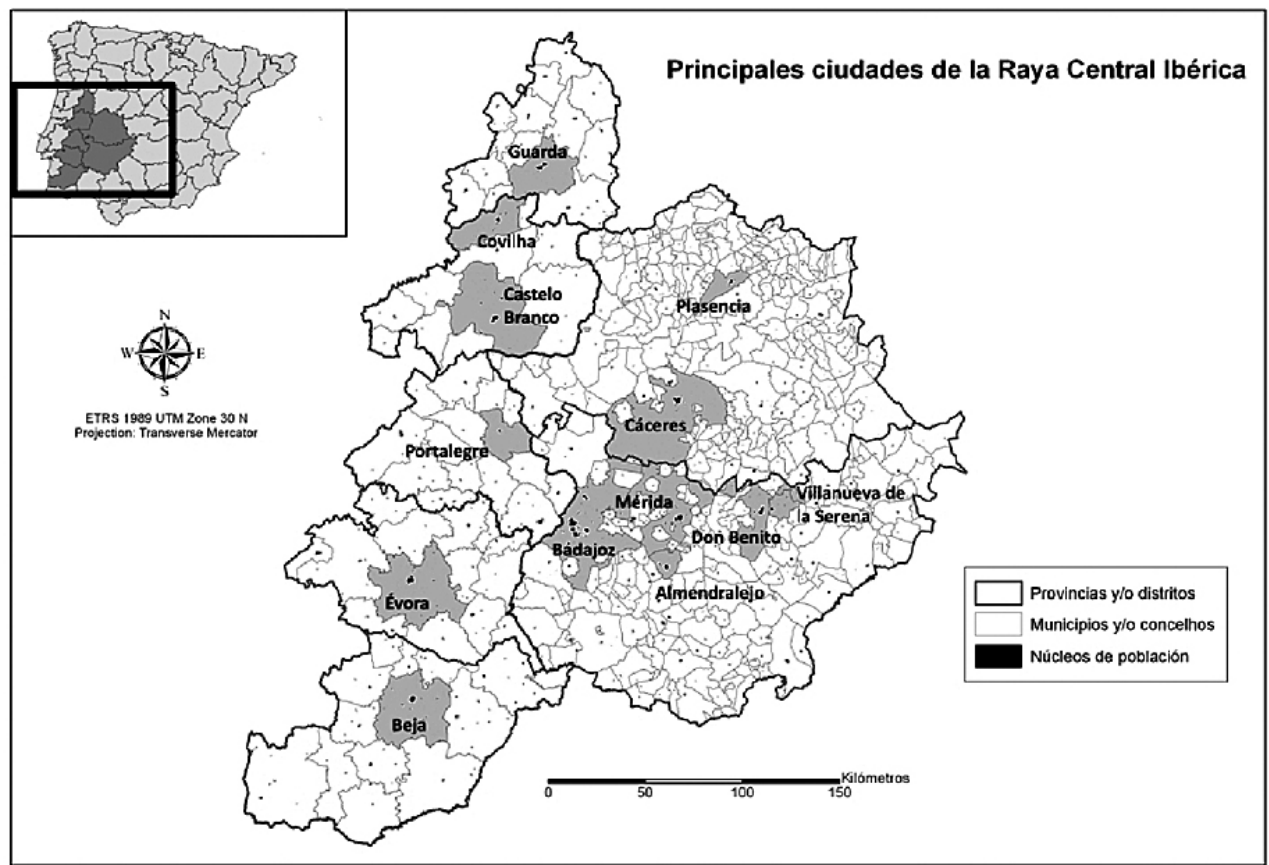

Fuentes: Instituto Nacional de Estadística de España: Divisiones municipales y provinciales e Instituto Geográfico Português: Carta Administrativa Oficial de Portugal. Elaboración propia 
En cuanto al medio físico o natural, concretamente la orografía, el espacio transfronterizo de la Raya Central Ibérica puede decirse que viene definido por tres aspectos fundamentales: el espacio montañoso que supone el Sistema Central en Extremadura y su prolongación en Portugal a través de la Serra da Malcata y la Serra da Estrela; las grandes penillanuras tanto en Extremadura (Penillanura Cacerena o La Serena) como en el territorio luso (Penillanura Alentejana); y las dos grandes cuencas hidrográficas de los ríos Tajo o Tejo y Guadiana. En la Figura 2, se muestra un mapa con los espacios más representativos del medio físico rayano.

Figura 2. Medio físico de la Raya Central Ibérica

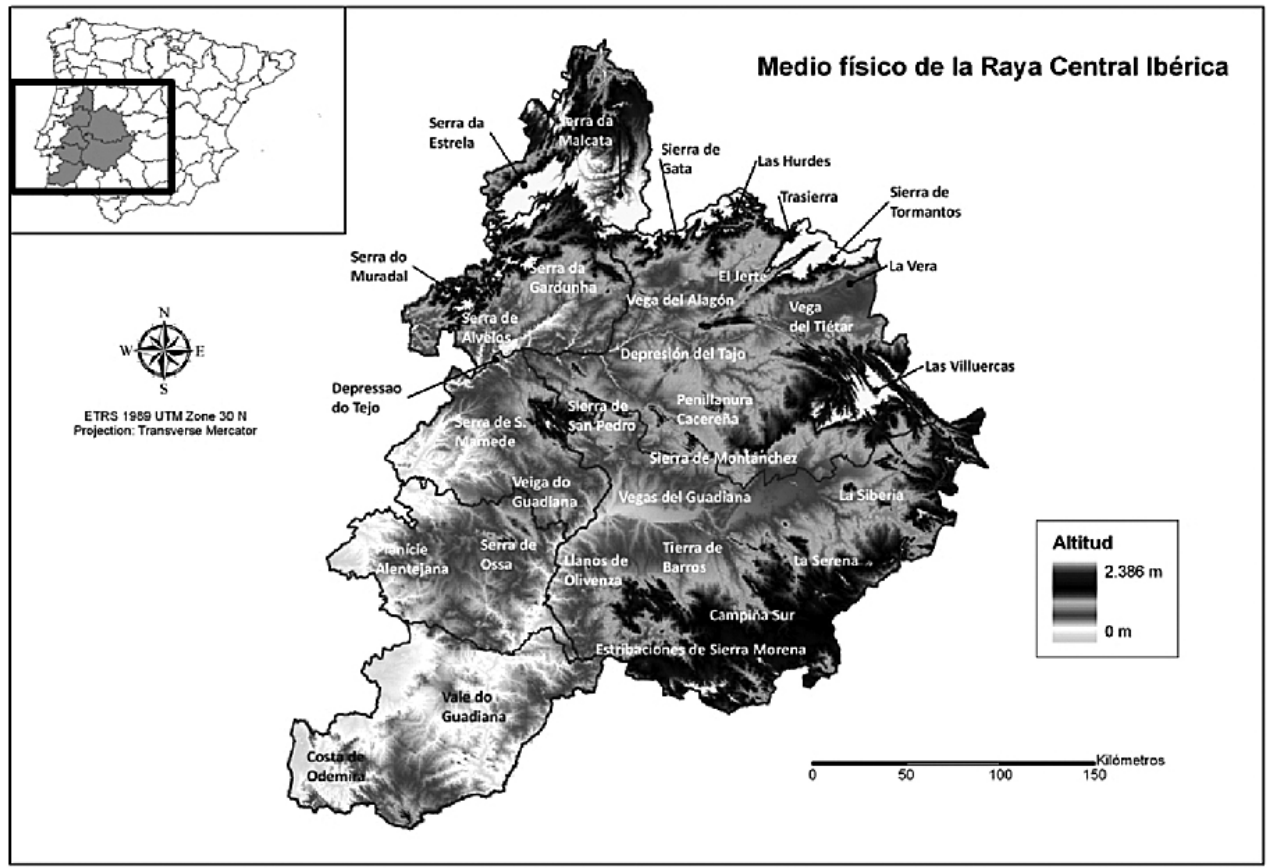

Fuente: Consortium for Spatial Information (CGIAR-CSI), NASA Shuttle Radar Topographic Mission (SRTM).Elaboración propia

\section{ESTADO DE LA CUESTIÓN. TÉCNICAS Y LÍNEAS DE INVESTIGA- CIÓN SEGUIDAS}

Diferentes estudios han tratado el tema de las dinámicas territoriales y sus repercusiones sobre los cambios de uso del suelo en espacios fronterizos desde varias perspectivas: desde aquellos que han estudiado el cambio demográfico y la dinámica territorial de forma genérica, hasta los que han investigado con mayor profundidad los cambios de usos del suelo y los impactos ambientales.

En el artículo, como se acaba de comentar, se analizan las nuevas dinámicas territoriales y sus repercusiones sobre los usos del suelo. Más concretamente, este estudio aborda los cambios de las superficies artificiales en los municipios y concelhos 
de la Raya Central Ibérica, tratando de dilucidar, en última instancia, qué variables independientes (socioeconómicas, agrarias, de accesibilidad o físicas) influyen en las mudanzas que afectan a la mencionada cobertura.

El área de estudio de este artículo, la Raya Central Ibérica, es un espacio transfronterizo marginal del suroeste europeo con una peculiaridades sociales, económicas, ambientales y culturales comunes, que han sido abordadas por diferentes investigadores.

En la actualidad, por primera vez en mucho tiempo, España y Portugal han emprendido una aventura conjunta que ha favorecido el abandono de viejos recelos y dotar a las relaciones políticas, sociales y económicas de un alto grado de cooperación. Ambos países tratan de demostrar su capacidad para enfrentarse solidariamente a los problemas similares, e incluso comunes, que les afectan (Cabero, 1997).

Respecto a la situación de la Raya Central Ibérica, en el interior peninsular, al factor de lejanía y de vacío demográfico se suma la debilidad de núcleos urbanos y semiurbanos, que apenas si muestran un comportamiento verdaderamente urbano (Campesino, 1997). El fortalecimiento de algunos ejes de comunicación junto con los nuevos comportamientos comerciales, supondría una desestructuración de las relaciones socioeconómicas en el interior transfronterizo. El escenario así analizado toparía con graves dificultades para llevar a cabo una ordenación y planificación territorial capaz de superar las carencias existentes.

Según el profesor Rocha (2008), tiene cada vez menos sentido hacer lecturas estrictamente nacionales de los sistemas urbanos y tratar de conocer si el desafío expresado en el primer INTERREG-A, con sus objetivos de contribuir para la fijación de la población y el crecimiento ordenados de los centros urbanos de dimensión media con importancia transfronteriza, han sido alcanzados. Así, pretende verificar si el proceso de transición de un modelo de centros urbanos dominantes hacia una red más equilibrada y policéntrica se está produciendo o no. La Raya Central Ibérica (Rocha, 2008) presenta, pues, algunas potencialidades morfológicas. Ésta precisaría reforzar las relaciones (flujos, redes, cooperación), para que sea posible transformar un sistema urbano excesivamente monocéntrico en otro más equilibrado y policéntrico.

\subsection{MODELOS ESTADÍSTICOS PARA EL ESTUDIO DE LOS CAMBIOS DE USO Y COBERTURA DEL SUELO}

Las cuestiones de cambio de uso del suelo y cambio en la cobertura del suelo han atraído el interés de una gran variedad de investigadores preocupados por la modelización de la conversión espacial y temporal de los patrones del suelo y por la comprensión de las causas y consecuencias de estos cambios (Irwin y Geoghegan, 2001). Entre estos, geógrafos y científicos expertos en ciencias naturales, han tomado la delantera en el desarrollo de modelos espacialmente explícitos de cambio de uso del suelo a una escala muy desagregada (por ejemplo, parcelas individuales de tierra o celdas de paisaje). Un significativo progreso se ha hecho en la adquisición de series de datos espaciales de sensores remotos (por ejemplo, imágenes de satélite de la cobertura del suelo), en la conceptualización de los procesos básicos geográficos y ambientales que están asociados con el cambio de uso del suelo y en el desarrollo de modelos espaciales que encajen con el proceso espacial de cambio de suelo razonablemente bien. 
Irwin y Geoghegan (2001) afirman que para la modelización de cambio de uso y cobertura del suelo es necesaria la aplicación de técnicas estadísticas espaciales para la elección de modelos discretos. Argumentan además que el desarrollo de mejores modelos económicos de cambio de uso y cobertura del suelo se apoya en los avances de la teoría económica espacial de estructura espacial urbana que puede explicar mejor los patrones espaciales temporales de migración, crecimiento de empleo, acciones de gobierno y resultado de los cambios de uso del suelo.

Según De Aranzábal et al. (2008), el paisaje cultural cambia porque la población que lo creó y lo ha mantenido históricamente está también modificando su sistema socioeconómico. Las estructuras territoriales y socioeconómicas mantienen una interacción constante y reciproca. Así, los procesos socioeconómicos son la principal causa de los cambios en los usos del suelo que, básicamente, determinan la estructura, la función y la dinámica de estos paisajes, por lo tanto los cambios en la estructura y procesos sociales llevan a una alteración del ambiente. Siguen para determinar esto a autores como Lorenzoni et al., 2000; Wang y Zhang, 2001; Wu y Hobbs, 2002.

Apuntan los autores que hay una ausencia de definiciones consistentes y métricas cuantificables respecto al papel jugado por los humanos en la modificación de los agro-ecosistemas. Siguiendo a Flamm y Turner (1994) afirman que modelar y simular los efectos de los factores humanos en los cambios en los paisajes permanece como un reto para la investigación ecológica, lo cual limita la integración de datos y modelos desde las ciencias naturales y sociales. Aseguran también que la información ecológica y socioeconómica debe ser integrada como base para la planificación y la gestión del suelo, tal y como ya habrían tratado Costanza y Voinov (2001), Matthews (2006) o Lacitignola et al. (2007). Por consiguiente, para estudiar la relación entre la estructura del paisaje y la socioeconomía subyacente emplean modelos numéricos que relacionan aspectos ecológicos y socio-culturales que permiten predecir nuevos tipos de paisaje para un escenario dado estableciendo cambios socioeconómicos, tal y como se ha realizado en la investigación. Los resultados contribuirían pues a establecer las posibles consecuencias en la naturaleza de cambios socioeconómicos generados por circunstancias exógenas (De Aranzábal et al., 2008).

Serra et al., (2008) desarrollan un análisis especial aplicado a determinar las principales fuerzas causantes de los cambios de cobertura y uso del suelo en una región mediterránea. Para ello utilizaron tres herramientas diferentes con el objeto de diferenciar los cambios de cobertura y uso del suelo, los factores condicionantes y las dinámicas del paisaje. Los cambios de cobertura y uso del suelo fueron cuantificados con técnicas de sensores remotos, los factores condicionantes se analizaron con regresiones logísticas múltiples, mientras que las dinámicas de los paisajes fueron cuantificadas usando diferentes estadísticas. En esta investigación de la Raya Central Ibérica se ha seguido varios estudios de Serra y para desarrollarla se han llevado a cabo los dos primeros análisis, estudio de los cambios y establecimientos de los factores condicionantes.

Las conclusiones del trabajo del autor catalán muestran que su área de estudio representa un buen ejemplo de los cambios de cobertura y uso del suelo en los paisajes mediterráneos europeos porque cubre tres diferentes emplazamientos socioeconómicos y ambientales. Mientras que alguna investigación ha analizado separada- 
mente ejemplos de cada ecoregión, una aproximación más integral ha permitido la comprensión de las consecuencias de los factores condicionantes en los tres diferentes escenarios (Serra et al., 2008). Las dinámicas de los paisajes y los impactos socioambientales que muestran las conclusiones del trabajo de Serra et al. también fueron muy interesantes: homogeneidad paisajística, incremento de los incencios forestales, mayor riesgo de erosión y degradación paisajística.

Se sigue también en esta investigación del espacio transfronterizo el trabajo de Dendoncker et al., (2007), en el cual los autores presentan un análisis espacialmente explícito de corte transversal de los factores que condicionan los usos del suelo. Muestran además que los modelos logísticos regresivos identifican tendencias o relaciones globales entre los factores socioeconómicos o físico-climáticos y la precisa localización de cada tipo de uso del suelo.

Para estos autores, cuando el objeto de un estudio es obtener el mejor modelo estadístico para comprender la distribución de los usos del suelo, simplemente un modelo autorregresivo puede ser apropiado. En el proyecto comparan tres tipos de modelos autorregresivos: (1) una serie de modelos de regresión logística binomiales explicando la proporción del uso del suelo modelado dentro de los alrededores de la celda; (2) una regresión autologística multinomial que explica la composición de los alrededores de la celda; y (3) un modelo basado en la Entropía Máxima Bayesiana que explica la organización espacial de los usos del suelo dentro del vecindario de una celda.

Los autores concluyen indicando que el modelo basado en la Entropía Máxima Bayesiana, no tiene ventajas sobre los otros métodos, teniendo en cuenta el área para la que ellos lo aplicaron. No obstante, también inciden en el hecho de que la explicación de la composición del vecindario de una celda sería esencial para obtener un encaje o ajuste más óptimo.

\section{MATERIAL Y MÉTODOS}

\subsection{DEFINICIÓN DE LAS VARIABLES SOCIOECONÓMICAS Y FÍSICAS}

La metodología seguida para este primer análisis socioeconómico y físico, que puede entenderse como estudio preliminar del área de estudio para su comprensión y la correcta selección de las variables independientes, es un asunto relevante. La correcta selección y el oportuno tratamiento de las variables explicativas son fundamentales para el correcto desarrollo de los modelos lineales generalizados. Así, para la selección de las variables independientes de entrada, se tuvo en cuenta la bibliografía consultada y los posibles casos de correlaciones y redundancias que pudieran darse. Se decidió que se realizaría un análisis descriptivo del área de estudio, para que estos conocimientos socioeconómicos y físicos, ayudaran a la comprensión de los fenómenos acaecidos en en el periodo de tiempo analizado.

En este estudio, tras la consulta de la bibliografía estudiada y la realización de diferentes análisis estadísticos previos con un conjunto de variables más numeroso (tratamiento de la colinealidad, correlación y homogeneidad), se emplearon definitivamente 30 variables independientes de entrada. 
La información alfanumérica usada en este primer análisis se obtuvo de diversas fuentes, en función del grupo de variables al que pertenecen. Comentar que se dividió en dos el análisis de las fuentes y definición y elaboración de las variables espaciales (por un lado las variables socioeconómicas, agrarias y físicas y, por otro, las de accesibilidad) por la gran diferencia existente tanto en el proceso de definición y elaboración como cartográfico. No obstante, recalcar que todas ellas constituyen un único grupo de variables independientes empleadas tanto para caracterizar el área de estudio como para calcular el modelo estadístico.

Así pues, se decidió emplear un total de 13 variables socioeconómicas (las variables sociales, demográficas, económicas y políticas, son las más importantes a la hora de provocar cambios, sobre todo cambios de superficies artificiales), 8 variables agrarias (por analizar un región transfronteriza rural en la que el sector primario todavía tiene mucha importancia: sistemas agroforestales, cultivos permanentes y terrenos regados permanentemente), 4 variables relacionadas con la accesibilidad (empleadas para ver como ha evolucionado la construcción de la infraestructuras del transporte y la accesibilidad en una región deprimida y marginal como es la estudiada) y por último cinco variables físicas (para determinar como influyen los factores físicos, climáticos y geomorfológicos sobre todo, en los cambios de cobertura y uso del suelo).

Por consiguiente, estas variables independientes, también denominadas variables explicativas son las que en el modelo lineal generalizado se utilizan para dar respuesta a los cambios de cobertura y uso del suelo que acontecen en el área de estudio en el periodo de tiempo analizado. Mencionar que, para el estudio planteado en este artículo, se decidió tomar en consideración a todos los municipios de las provincias de Cáceres y Badajoz, así como a los concelhos de los distritos portugueses fronterizos con Extremadura de Guarda, Castelo Branco, Portalegre, Evora, y Beja. De este modo se realizaba una comparación de unas unidades administrativas más homogéneas, sin obviar que en Portugal éstas son mucho más extensas.

\subsection{OCUPACIÓN Y CAMBIOS DE COBERTURA Y USO DEL SUELO: LAS SUPERFICIES ARTIFICIALES}

Para el estudio de la ocupación y los cambios de cobertura y uso del suelo se han empleado los datos aportados por el Programa CORINE. Este programa se inicia en virtud de una decisión del Consejo de Ministros de la Unión Europea (CE/338/85), pasando en 1995 a ser responsabilidad de la Agencia Europea de Medioambiente (AEMA). Su finalidad era la creación y actualización permanente de una base de datos sobre los usos del suelo del territorio europeo.

El Instituto Geográfico Nacional de España y el Instituto do Ambiente e Instituto Geográfico Português fueron los encargados, entre 1987 y 1991, de coordinar la ejecución del CORINE Land Cover 1990 (CLC 90) en España y Portugal, bajo la supervisión de la Comisión Europea. La Agencia Europea de Medio Ambiente inició a finales de los años noventa una actualización con el objeto de proveer la información necesaria y oportuna para la obtención de los indicadores de cambios en las tendencias de ocupación del suelo. El nuevo proyecto tenía dos componentes principales 
interconectadas. La primera de ellas, Image 2000 y la la segunda, CORINE Land Cover 2000 (CLC 2000). El último proyecto CORINE Land Cover data de 2006, apareciendo publicado en Portugal en el año 2009 y en España en marzo del año 2010.

Como ya se ha comentado, en este estudio de las superficies artificiales, se han llevado a cabo dos análisis independientes: en el primero se tratan los principales intercambios de cobertura del suelo de Nivel 1 del proyecto CLC, en los que interviene la cobertura de las superficies artificiales; y el segundo es un análisis del cambio neto de la superficie artificial acontecido en el área de estudio en el periodo de tiempo estudiado.

Para el tratamiento de los intercambios de cobertura del suelo se deben seguir diferentes pasos. El primer paso es calcular a nivel municipal la superficie ocupada por cada uno de los usos del suelo para el Nivel 1 empleado. Acto seguido, se tratá de ver, dentro de cada municipio/concelho, los intercambios de cobertura y uso del suelo que se han producido entre un tipo de uso y otro. Para ello se realizaron un gran número de operaciones con el Sistema de Información Geográfica empleado (ArcGIS 9.2.), como de consultas en Access, las cuales daban como resultado un valor para cada uno de los municipios/concelhos (identificados siempre por su Código INE).

Para la realización del segundo análisis, a saber, el estudio de los cambios netos de superficie (en este caso cobertura artificial) a nivel municipal, se necesita las diferentes operaciones y consultas del análisis anterior que dan como resultado una tabla. Esta debe exportarse a Excel y, allí, se ejecutan las diferentes operaciones estadísticas requeridas para obtener los cambios netos de superficie. Tras la obtención del cambio neto de la superficie artificial, la tabla resultante se importa a $A r c G I S$ para su correcta manipulación y realización de la cartografía de resultados.

\subsection{MODELO LINEAL GENERALIZADO}

El objetivo último del trabajo que se presenta no es tan sólo analizar la ocupación de las superficies artificiales, los intercambios de cobertura en los que se ven afectados o los cambios netos de este uso en el periodo de tiempo tratado. La finalidad es también, llegar a conocer cuáles son los factores (en forma de variables socioeconómicas, agrarias, de accesibilidad o geofísicas) que condicionan las modificaciones que han padecido las superficies artificiales desde 1990 a 2000.

Tras experiencias previas con diferentes técnicas y modelos estadísticos (SOMF, Mars,...) y tras la lectura de una extesa bibliografía, se decidió emplear la regresión logística como técnica para explicar los cambios de cobertura del suelo. Más concretamente, lo que se usaron fueron modelos lineales generalizados o Generalized Linear Models (GLM), concretamente la función $0 \mathrm{glm}$ del programa estadístico $R^{4}$.

\footnotetext{
${ }^{4} \mathrm{R}$ es un sistema para la implementación de funciones estadísticas y la creación de gráficos. En un sentido más amplio, $\mathrm{R}$ se considera en sí mismo un lenguaje de programación con un conjunto de procedimientos implementados que permiten realizar tareas específicas muy diversas, que van desde la aplicación de funciones estadísticas a la generación y resolución de múltiples problemáticas (Crawley, 2005; Crawley, 2007)
} 
Los modelos lineales se encuadran dentro de los análisis de regresión. Sin entrar en muchos detalles, cabe recordar que los modelos lineales se basan en una serie de supuestos, algunos de los cuales pueden y deben comprobarse una vez ajustado el modelo. Estos son: independencia, linealidad, normalidad u homocedasticidad. Pues bien, en muchas ocasiones, sin embargo, uno o varios de estos supuestos no se cumplen. Por ejemplo, en algún caso concreto puede suceder que a medida que aumenta la media de la muestra, aumente también su varianza. Estos problemas se pueden llegar a solucionar mediante la transformación de la variable respuesta (tomando logaritmos). Sin embargo, estas transformaciones no siempre consiguen corregir la falta de normalidad, la heterocedasticidad o la no linealidad de los datos $^{5}$. Además resulta muchas veces difícil interpretar los resultados obtenidos. Una alternativa a la transformación de la variable respuesta y a la falta de normalidad es el uso de los modelos lineales generalizados. Los modelos lineales generalizados son una extensión de los modelos lineales que permiten utilizar distribuciones no normales de los errores (binomiales, Poisson, gamma, etc.) y varianzas no constantes (LE SAGE ET AL. 2009).

Ciertos tipos de variables respuestas sufren invariablemente la violación de estos dos supuestos de los modelos normales y los GLM ofrecen una buena alternativa para tratarlos. Específicamente, se puede considerar utilizar GLM cuando la variable respuesta es: un conteo de caso, un conteo de casos expresados como proporciones, o una respuesta binaria. En este artículo, se está ante una respuesta binaria: aumento de la superficie artificial (1) y disminución o mantenimiento de la superficie artificial (0); por lo tanto, el uso de GLM está justificado.

La reducción del incremento de las superficies artificiales a una variables binario o dicotómica (aumenta/se reduce o mantiene) puede hacer que se pierda información en los análisis estadísticos, pero la gran varianza explicada por el modelo $(42,52 \%)$ teniendo en cuenta que hablamos de una variable dependiente dicotómica es muy significativa. Además hay que subrayar que esta técnica de los modelos lineales generalizados ha sido la única que ha conseguido explicar los factores causantes de los cambios de coberturas y usos del suelo en la Raya Central Ibérica, teniendo en cuenta la particularides de las variables independientes y las variables respuestas.

\section{RESULTADOS}

\subsection{INTERCAMBIOS DE COBERTURAS EN LA RAYA CENTRAL IBÉRICA}

En este punto, se expone los principales intercambios de cobertura que se han producido en los municipios o concelhos del área de estudio, para el periodo de 1990 a 2000, centrando el análisis en aquellos más significativos que afectan a las superficies artificiales. Por consiguiente, se tratan aquellas coberturas que poseen tanto una media relevante, como unos valores máximos que merece la pena ser comentados.

5 Tampoco puede silenciarse uno de los problemas más importantes en el ámbito espacial: el fenómeno de la autocorrelación espacial, que puede producirse también en los residuos de una regresión. 
Figura 3. Intercambios de zona agrícola a superficie artificial

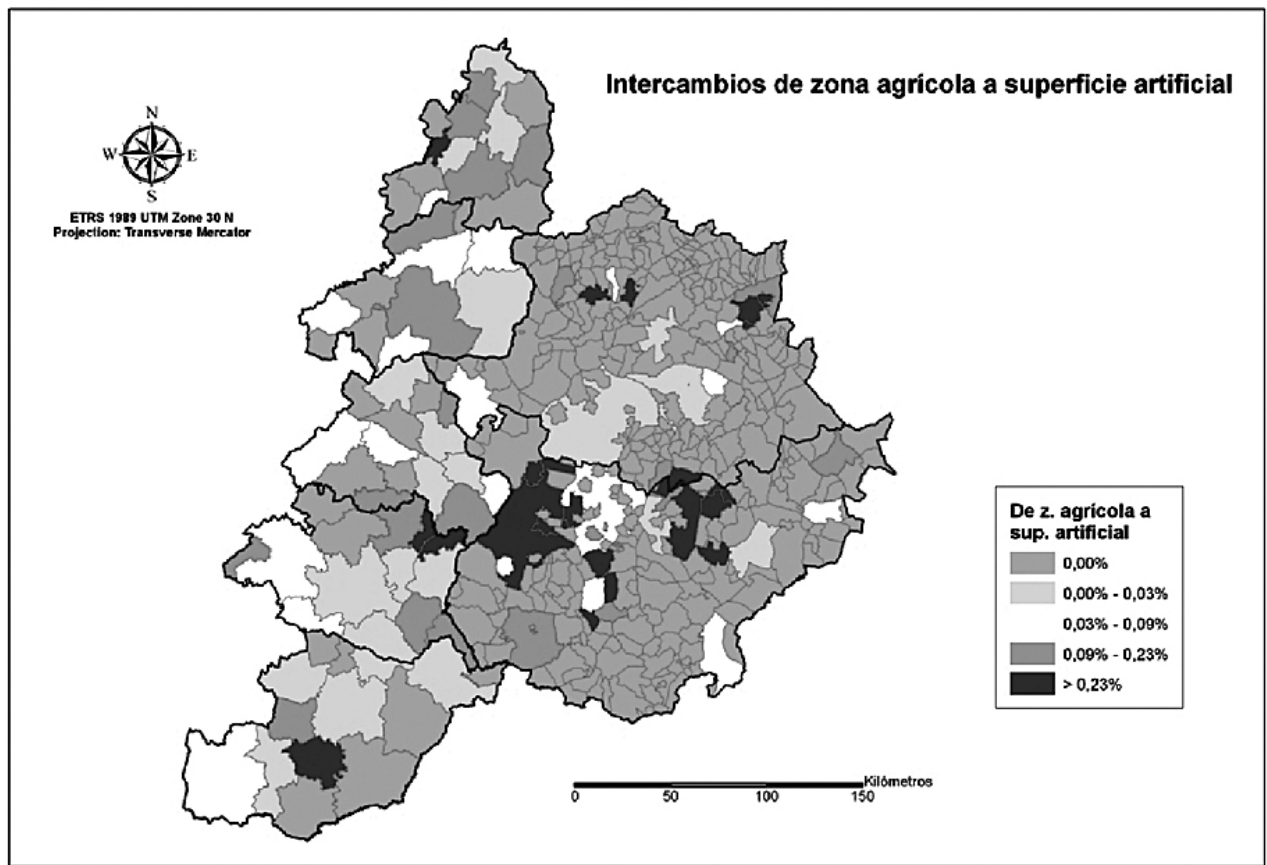

Fuentes: CORINE Land Cover - Proyecto I\&CLC2000 - España (IGN, CNIG) y CORINE Land Cover - Projecto I\&CLC2000 - Portugal (IA e IGP). Elaboración propia

El primero de los intercambios y con una media municipal del $0,03 \%$, es el que va de las zonas agrícolas a las superficies artificiales (ver Figura 3). Si se considera la media de España $(0,34 \%)$ y la de Portugal $(0,47 \%)$, está claro que este fenómeno, o este flujo de cobertura es menos importante en la Raya Central. Si se atiende al mapa, parece evidente que los municipios o concelhos en los que aumenta en mayor medida la superficie artificial procedente de zonas agrícolas, son los que poseen una mayor entidad poblacional o lo que disfrutan de un mayor dinamismo demográfico y económico. Este hecho se hace patente tanto en los distritos portugueses como en las provincias extremeñas.

Tras el análisis municipal de los intercambios de zonas agrícolas a superficies artificiales, se procede al estudio del flujo de cobertura de Nivel 1 que se produce desde las zonas forestales a las superficies artificiales (ver Figura 4). En esta ocasión, el predominio de los términos que no han sufrido un intercambio de este tipo, es aún más evidente que en la variable anterior. Nuevamente se aprecia con claridad como son los municipios más poblados o dinámicos (agricultura, industria o comercio) los que sufren mayores transformaciones. También destacan aquellos que tiene dentro de sus límites alguna explotación minera. No obstante, la media de intercambio a nivel municipal vuelve a ser muy reducida $(0,02 \%)$, lo que hace que esta variable tan sólo será realmente significativa en los municipios con valores máximos. Al igual que en el caso anterior, la media del territorio español $(0,14 \%)$ y portugués $(0,26 \%)$ es superior a la del área de estudio. 
Figura 4. Cambios de zona forestal a superficie artificial

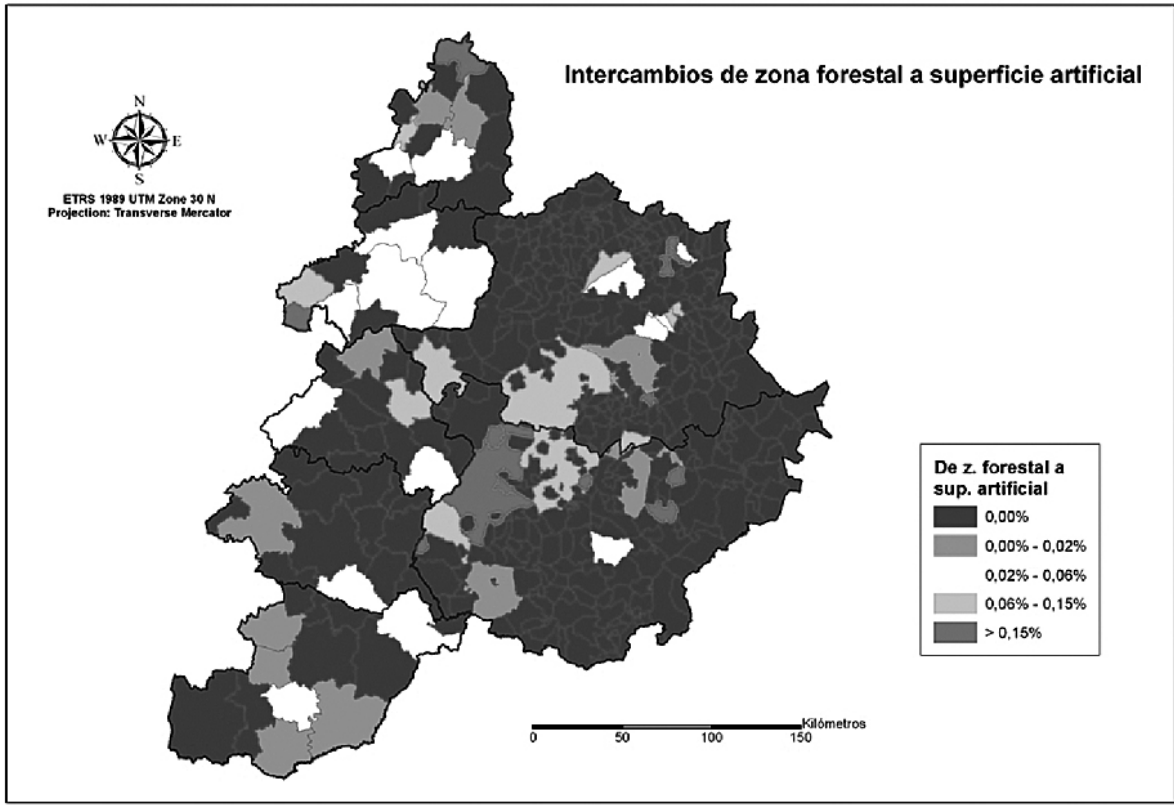

Fuentes: CORINE Land Cover - Proyecto I\&CLC2000 - España (IGN, CNIG) y CORINE Land Cover - Projecto I\&CLC2000 - Portugal (IA e IGP). Elaboración propia

\subsection{CAMBIOS NETOS DE SUPERFICIE EN LAS COBERTURAS ARTIFICIALES}

Una vez se han analizado los intercambios más relevantes que afectan a las superficies artificiales, a continuación se expone el cambio neto de la cobertura artificial de Nivel 1 que ofrece el CORINE Land Cover.

El cambio neto de las superficies artificiales (ver Figura 5) dibuja un mapa para la Raya Central Ibérica muy significativo. En él se aprecia con total claridad cuales son los municipios o concelhos en los que las superficies artificiales aumentan en mayor medida, y que por regla general, suelen coincidir con los núcleos más poblados y dinámicos socioeconómicamente hablando. No obstante, hay que subrayar que el crecimiento medio de las superficies artificiales en la Raya Central Ibérica es tan sólo del $0,05 \%$. Este es un porcentaje muy reducido si se tiene en cuenta el valor medio de España $(0,47 \%)$ y de Portugal $(0,79 \%)$.

Normalmente, el aumento de la superficie artificial, tiene lugar en aquellos municipios o concelhos con un mayor dinamismo demográfico y económico tanto en territorio luso como en territorio extremeño. No obstante, conviene resaltar el hecho de que en los distritos portugueses, son apenas 16 los concelhos en los que no se han producido cambios en esta cobertura del suelo. El predominio de aquellos en los que esta superficie aumenta, sin duda también viene determinado por la estructura administrativa y poblacional que presentan el estado luso, y donde destaca la población diseminada. Por su parte, los municipios extremeños con un cambio neto positivo, si que coinciden más claramente, con los más poblados y desarrollados de la región. Sólo dos 
municipios, ubicados en la comarca natural de los Montes del Guadiana en Badajos, ven como su superficie artificial disminuye en el periodo de tiempo estudiado.

Figura 5. Cambio neto porcentual en las superficies artificiales

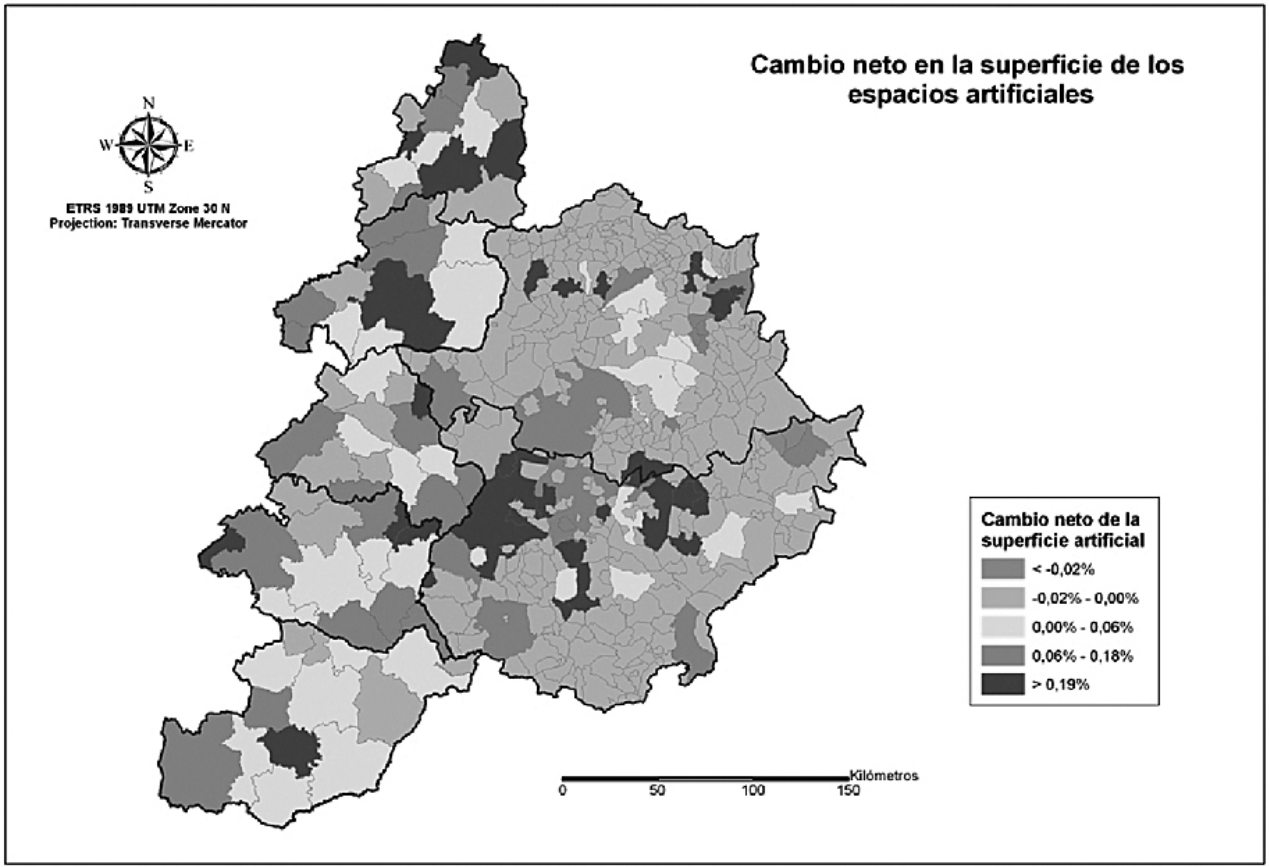

Fuentes: CORINE Land Cover - Proyecto I\&CLC2000 - España (IGN, CNIG) y CORINE Land Cover - Projecto I\&CLC2000 - Portugal (IA e IGP). Elaboración propia

\subsection{FACTORES DETERMINANTES DE LOS CAMBIOS DE COBERTURA}

Tras el análisis de la situación y la evolución de las superficies artificiales en el área de estudio en el periodo de tiempo tratado (1990-2000), se está en disposición de realizar el modelo estadístico que ayude a comprender la realidad de las superficies artificiales en la Raya Central.

En este epígrafe pues, se exponen el resultado del GLM de la variable dependiente que supone las superficies artificiales. En un GLM, todas aquellas variables dependentes que poseen una devianza ${ }^{6}$ superior al $20,00 \%$, se consideran significativas

${ }^{6}$ La cantidad de varianza explicada por el modelo se conoce en los GLM como devianza. La devianza da una idea de la variabilidad de los datos. Por ello, para obtener una medida de la variabilidad explicada por el modelo, se ha de comparar la devianza del modelo nulo (Null deviance) con la devianza residual (Residual deviance), esto es, una medida de cuánto de la variabilidad de la variable respuesta no es explicado por el modelo.

Por tanto, la devianza o varianza explicada por el modelo se obtendría de:

D2=((Devianza.modelo.nulo-Devianza.residual)/Devianza.modelo.nulo $)^{*} 100$ 
para el análisis que se ha llevado a cabo. Por consiguiente, como en el caso de las superficies artificiales, la devianza es del $42,52 \%$, se puede decir que las variables independientes empleadas en el modelo, explican muy bien los cambios acontecidos con esta cobertura. En este caso, para la correcta interpretación de los resultados del modelo calculado para la variable dependiente tratada una tabla con los resultados del modelo y los gráficos de caja de las variables que más explican la variable dependente. Hay que recordar también que para el cálculo del modelo, ya que en el GLM aplicado la variable dependiente debe ser dicotómica, se asignó un 1 a todos los municipios en los que la cobertura del suelo aumentaba, mientras que con un 0 se caracterizaba a aquellos en los que la cobertura disminuía o permanecía estable.

Tabla 1. Resultados del modelo estadístico extraído para la variable dependiente: superficie artificial del CLC nivel 1

\begin{tabular}{|l|r|r|r|r|r|}
\hline \multicolumn{7}{|c|}{ Modelo estadístico extraído para la superficie artificial } \\
\hline & Estimate & Std. Error & $\mathrm{z}$ value & $\operatorname{Pr}(>|\mathrm{z}|)$ & \\
\hline (Intercept) & $-1,096$ & 0,180 & $-6,104$ & 0,000 & $* * *$ \\
\hline Población total & 1,960 & 0,245 & 7,992 & 0,000 & $* * *$ \\
\hline Tasa de natalidad & 0,813 & 0,360 & 2,262 & 0,024 & $*$ \\
\hline Densidad de ganado porcino & $-0,272$ & 0,137 & $-1,991$ & 0,047 & $*$ \\
\hline $\begin{array}{l}\text { Tiempo mínimo de acceso a los centros } \\
\text { de actividad económica de la Raya Central }\end{array}$ & 0,966 & 0,438 & 2,204 & 0,027 & $*$ \\
\hline Distancia a las principales vías de comunicación & $-0,721$ & 0,268 & $-2,691$ & 0,007 & $* *$ \\
\hline Altitud media & $-1,372$ & 0,465 & $-2,949$ & 0,003 & $* *$ \\
\hline
\end{tabular}

Fuentes: CORINE Land Cover - Proyecto I\&CLC2000 - España (IGN, CNIG) y CORINE Land Cover - Projecto I\&CLC2000 - Portugal (IA e IGP). Elaboración propia

La Tabla 1 muestra el modelo estadístico extraído para la variable dependiente de Nivel 1 de las superficies artificiales. Todas las variables independientes cuentan con un $\operatorname{Pr}(>|z|)$ inferior a 0,05 , por lo que todas ellas son relevantes a la hora de explicar los cambios. Según el resultado del modelo lineal generalizado, las variables explicativas de aumento de la superficie artificial, por orden de relevancia son: la población total, la altitud media, la distancia a las principales vías de comunicación, la tasa de natalidad, el tiempo mínimo de acceso a los centros de actividad de la Raya Central Ibérica y la densidad de ganado porcino.

Para explicar mejor el resultado del modelo, se utilizan diagramas de cajas para representar las variables explicativas. Estos gráficos de caja sirven para dilucidar la distribución de los valores respecto a la media ${ }^{7}$. Lo que se pretende con estos gráficos es ver como se comporta la variable dependiente o dicotómica, en función de cada una de las variables independientes. Por consiguiente, se trata de comprender como afecta o influye una determinada variable socioeconómica o física, en el aumento (1) o disminución (0) de una

${ }^{7}$ En los diagramas de cajas la media es la línea negra que se encuentra dentro de la caja. Fuera de la caja aparecen otras dos líneas que representan la desviación estándar. La caja es más ancha o menos ancha en función de la dispersión de los datos respecto a la media. Los puntos que están fuera de la caja y las líneas son los máximos y los mínimos fuera de los rangos definidos. 
cobertura o uso del suelo en cuestión. Por supuesto, también es muy importante ver la distribución de los valores respecto a la media y la existencia o no de valores extremos.

La Figura 6 expone los diagramas de caja de todas las variables independientes que intervienen en los cambios de las superficies artificiales. El primero de los gráficos muestra la influencia de la población total de los municipios o concelhos en los cambio de esta cobertura. No hay duda de que la superficie artificial aumenta en mayor medida cuando la población de los términos es más importante. Pese a esto, conviene destacar la presencia de algunos valores extremos tanto en el caso de los municipios que aumentan su superficie, como en aquellos que no cambian esta cobertura tratada.

Figura 6. Representación gráfica de la relación de la variable dependiente con las variables independientes más significativas
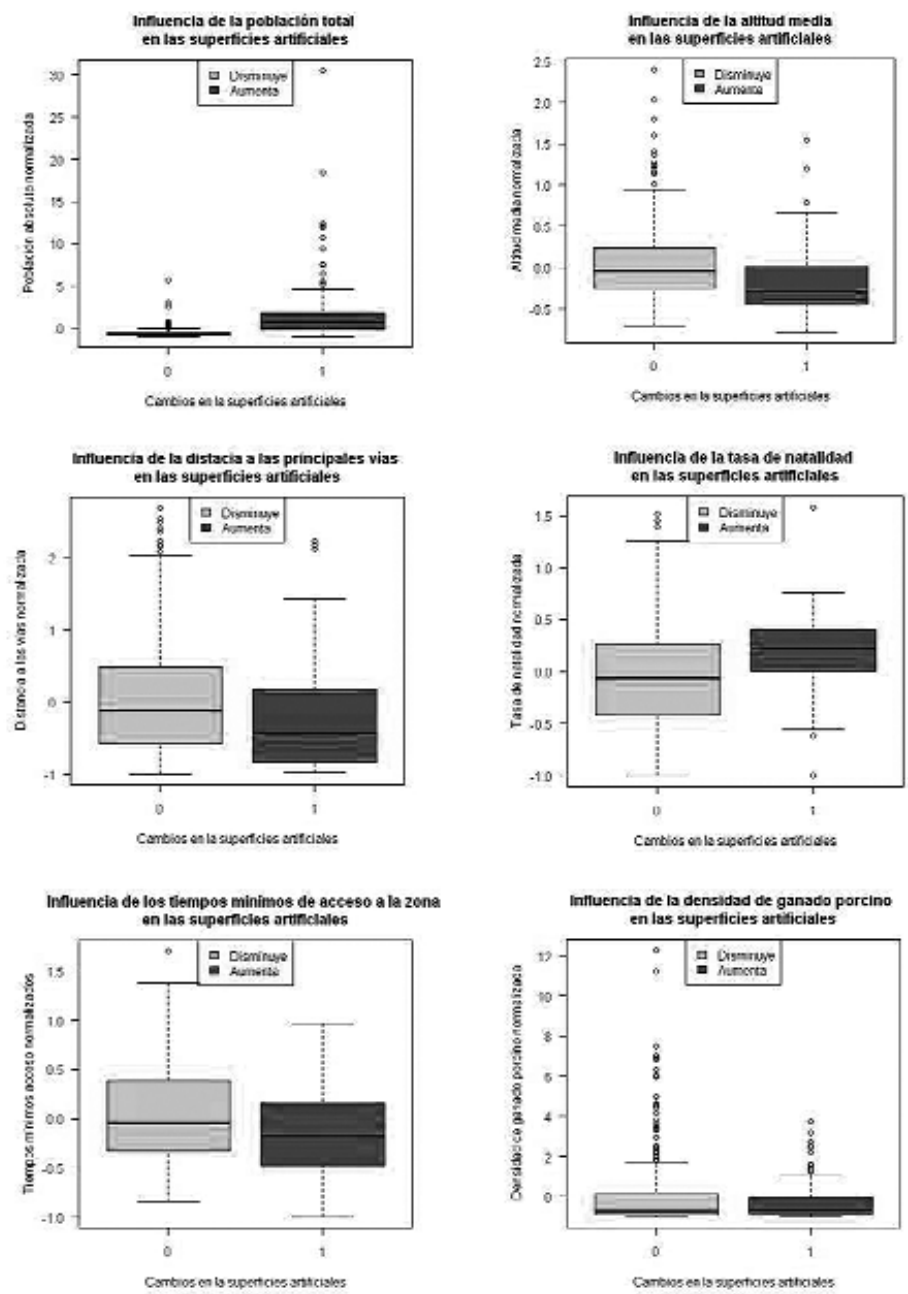

Fuentes: CORINE Land Cover - Proyecto I\&CLC2000 - España (IGN, CNIG) y CORINE Land Cover - Projecto I\&CLC2000 - Portugal (IA e IGP). Elaboración propia 
Puede sorprender la gran relevancia que muestra el modelo para la variable independiente de la altitud, pero la relación entre la altitud y la presencia de población o ubicación de ciudades o no, es un hecho. Por regla general, los asentamientos poblacionales se han situado siempre en zonas con una reducida altitud, más concretamente, en cuencas sedimentarias o zonas de vega, ya que son las más productivas a la hora de conseguir alimento. Así pues, el hecho de que la superficie artificial aumente más cuando la altitud sea menor, es una circunstancia no sólo relevante, sino además, coherente.

La relación existente entre las infraestructuras del transporte y el desarrollo demográfico y económico de una zona está ampliamente demostrada. Por lo tanto, es lógico que la cobertura tratada aumente en las zonas situadas más próximas a las principales vías de comunicación, ya que la presencia de grandes infraestructuras motiva el desarrollo industrial, el del sector servicios y, por ende, el crecimiento poblacional, con lo que esto conlleva.

La alta tasa de natalidad, asociada en gran medida al desarrollo económico, y por consiguiente a las aglomeraciones poblacionales, está directamente relacionada con el desarrollo de las superficies artificiales.

El gráfico muestra como el aumento de la superficie artificial es mayor en aquellos municipios más próximos, o con menor tiempo de acceso, a los centros de actividad económica de la Raya Central Ibérica; esto quiere decir que los espacios artificializados aumentarán allí donde el desarrollo económico es mayor.

\section{DISCUSIÓN}

El estudio ha demostrado la idoneidad de integrar factores humanos y biofísicos en la modelización de los cambios de cobertura y uso del suelo. La detección y el análisis de los cambios de cobertura y uso del suelo ha sido aplicada satisfactoriamente en países y ecosistemas muy diferentes de todo el mundo. Diferentes enfoques de modelización han sido usados para comprender dónde están ocurriendo los cambios de cobertura y uso del suelo y para estudiar los factores que condicionan estos cambios.

En la actualidad, en muchas partes del mundo, la actividad humana es el principal factor a la hora de provocar cambios de coberturas y usos del suelo, aunque la estructura física subyacente del paisaje puede modificar la cobertura y uso del suelo. Por ejemplo, las condiciones del suelo o las pendientes del terreno pueden hacer el cultivo de algunos cereales difícil. Por lo tanto, una integración de factores biofísicos y humanos en la explicación de las dinámicas de la cobertura y uso del suelo queda como una importante tarea de investigación (Serra et al., 2008).

Aún reconociendo la importancia de los factores físicos, esto no excluye que los factores socioeconómicos lleguen a ser la principal influencia en las decisiones de los titulares de las explotaciones respecto a los usos del suelo, por ejemplo. Por esta razón estos son, en general, los factores condicionantes más relevantes de los cambios de cobertura y uso del suelo.

Un aspecto sobre el que merece la pena discutir, teniendo en cuenta los resultados de la caracterización socioeconómica y la importancia de las variables relacionadas 
con la accesibilidad en el modelo lineal generalizado, es la necesidad de completar la red de infraestructuras en la Raya Central Ibérica. Todo el territorio eminentemente transfronterizo es el que peor accesibilidad presenta en el área de estudio, posiblemente fruto de ser una zona periférica y por la ausencia de grandes ciudades. No obstante, conviene destacar también la situación del este de la región extremeña, donde sus comarcas funcionales y/o naturales, presentan una situación marginal, no sólo respecto a los grandes centros de actividad de la Península, sino también, por su lejanía a los grandes núcleos del área de estudio. Esta situación periférica, la falta de accesibilidad y la carencia de buenas infraestructuras, está llevando a que las necesidades de acceso a los bienes, servicios y equipamientos, motiven el abandono poblacional de estos lugares por parte de la población más joven y a que se acreciente el problema del envejecimiento, todo ello con sus implicaciones sociales, económicas y ambientales.

Para corregir las disparidades en una región transfronteriza tan extensa y con una población tan dispersa, es necesario acometer una auténtica ordenación territorial en la Raya Central Ibérica, donde se concrete el papel del mundo rural, la cooperación urbano-rural y el desarrollo policéntrico (siguiendo la Estrategia Territorial Europea). En la actualidad la ordenación territorial no existe en el área de estudio, porque no se han redactado instrumentos básicos que aborden la cuestión y tracen el modelo de organización territorial para el presente y el futuro próximo y porque no hay una política que priorice las medidas tendentes a impedir la concentración de la economía en pocos núcleos urbanos que están absorbiendo la población y el sistema económico. Estos reciben políticas e inversiones de preferente localización industrial, planes regables, centros hospitalarios, educativos, universitarios, etc., tanto con fondos nacionales como comunitarios; presentan a su vez, la mayor accesibilidad por la disposición de la red de infraestructura de comunicaciones. Mientras, el resto del territorio pierde población. Son muchas las debilidades de este espacio y de distinto carácter, pero también cuenta con grandes fortalezas. La principal de todas ellas es el propio territorio, teniendo en cuentan el excelente grado de conservación del medio ambiente, avalado todo por los contrastes climáticos, edáficos e incluso biogeográficos.

\section{CONCLUSIONES}

La principal conclusión que podemos extraer es que el crecimiento de las superficies artificiales, significativo porcentualmente y poco relevante superficiealmente, se sigue produciendo en la Raya Central Ibérica pese a la baja densidad y la débil estructura urbana. Dentro de las zonas urbanas, el tejido urbano discontinuo presenta una mayor superficie, motivado quizá por la importancia que la población diseminada tiene en el territorio portugués y por el crecimiento de un modo difuso que está teniendo lugar en las principales ciudades. Las zonas industriales o comerciales es la segunda tipología de superficie artificial con mayor importancia superficial en el territorio estudiado. La Raya Central ha sufrido un cambio muy importante en lo que se refiere a las redes de transporte por carretera, principalmente, lo que ha supuesto un salto de calidad muy importante en las mismas. 
Un resultado muy importante del estudio que hay que subrayar es el proceso de abandono del medio rural y concentración de la población en las grandes ciudades. Esta situación actual de la Raya Central Ibérica, por la que se deteriora todo el medio físico y natural, está motivada por un modelo territorial carente de sentido tanto en el territorio portugués (dispersión de la población, excesivo número de núcleos, multiplicación de equipamientos y servicios, concentración de la población en el litoral), como en el espacio extremeño (concentración de la población en las principales ciudades y abandono rural, envejecimiento de la población). En el caso de los distritos portugueses, puede que la presencia de algunos concelhos con una población considerable y con núcleos importantes, estén conteniendo el problema. No obstante, lo que no puede obviarse es que los concelhos rurales del interior, sin duda en periodo de despoblación, con un gran problema de envejecimiento y con un sistema socioeconómico marginal, son un gran inconveniente para el desarrollo territorial de este espacio, además del punto del que parten todos los problemas que motivan los cambios de coberturas y usos de suelo y el abandono del medio (incendios forestales o invasiones de especies). En el espacio extremeño, la gran distancia que separa a cantidad de núcleos rurales y semirrurales de las principales ciudades regionales e incluso nacionales, unido a un sistema público de transporte caracterizado por la escasez de servicios y malos horarios, está llevando a la creación de espacios y núcleos de población es una situación marginal y remota. La debilidad del sistema urbano para articular un territorio tan amplio, por consiguiente, parece que está llevando al abandono de los núcleos rurales ante la falta de oportunidades y su desplazamiento hacia ciudades que les ofrezcan mayores oportunidades.

En cuanto a la metodología seguida en el trabajo de investigación, destacar que el tratamiento de las superficies artificiales en la Raya Central Ibérica sirve para su propósito final: definir un proceso metodológico que ayude al estudio de los cambios de cobertura y uso del suelo y a la definición de los factores causantes de los cambios de las superficies. En primer lugar, los resultados del análisis, muestran que la bibliografía consultada para la elección de las variables independientes o explicativas y las técnicas para su tratamiento, son correctas. Las variables socioeconómicas, agrícolas, de accesibilidad y físicas, sirven para explicar los cambios de las superficies artificiales. Hay que destacar sobre todo la coincidencia entre gran parte de las variables físicas y de accesibilidad que se emplean aquí, y las usadas por otros investigadores. En segundo lugar, este estudio ha servido para confirmar la idoneidad del empleo de los modelos lineales generalizados para el estudio de los factores condicionantes de los cambios de cobertura o uso del suelo.

Junto a estos aspectos, otros aspecto metodológicos abordados con éxito en el trabajo y que se han aplicado satisfactoriamente en la Raya Central Ibérica son: la necesidad de una correcta selección de las variables independientes, evitando en todo momento la temida correlación entre ellas que pudiera alterar los resultados del modelo; y las dudas que se planteablan con la oportunidad o no de emplear modelos que tuvieran en cuenta la autocorrelación espacial, las cuales fueron aclaradas tras los nulos resultados que se obtuvieron para el área de estudio, y con las conclusiones de otros trabajos. 


\section{AGRADECIMIENTOS}

Agradecer el apoyo institucional prestado por el Ministerio de Educación de España. Los autores agradecen de igual modo al Ministerio de Educación la Beca FPU 2006-03185 y a la Universidad de Extremadura, concretamente la Facultad de Filosofía y Letras, el uso de sus instalaciones y equipamientos.

\section{REFERENCIAS BIBLIOGRÁFICAS}

CABERO, V.: Portugal y España: Una mirada geográfica a las relaciones ibéricas, Boletín de la Asociación de Geógrafos Españoles, 25, 1997, 3-13.

CAMPESINO, A. J., CABERO, V.: Knowledge of border areas: the contribution of Spanish geographers, Boletín de la Asociación de Geógrafos Españoles, 21-22, 1995-1996, 83-98.

CAMPESINO, A. J.: Territorio y ciudades abaluartadas en la Raya / Raia Ibérica: De frontera a patrimonio mundial en serie. En Cruz, M. (coord.) (2007): Ciudades y núcleos fortificados de la frontera hispano-lusa: el territorio de Extremadura y Alentejo. Historia y patrimonio, Universidad de Extremadura, Servicios de Publicaciones, pp. 257-268.

CAYETANO, M.: Extremadura y Alentejo: Del subdesarrollo heredado a los retos del futuro, Revista de Estudios Extremeños, 62, 2006, 1167-1188.

COSTANZA, R., VOINOV, A.: Modelling ecological and economic systems with stela: Part III". En De Aranzábal, I., Schmitz, M. F., Aguilera, P., Pineda, F. D.: Modelling of landscape changes derived from the dynamics of socio-ecological systems. A case of study in a semiarid mediterranean landscape, Ecological Indicators, 8, 2008, 672-685.

CRAWLEY, M. J. (2005): Statistics. An introduction using R, Wiley.

CRAWLEY, M. J. (2007): The R book, Wiley.

DE ARANZÁBAL, I., SCHMITZ, M. F., AGUILERA, P., PINEDA, F. D.: Modelling of landscape changes derived from the dynamics of socio-ecological systems. a case of study in a semiarid mediterranean landscape, Ecological Indicators, 8, 2008, 672-685.

DENDONCKER, N., ROUNSEVELL, M., BOGAERT, P.: Spatial analysis and modelling of land use distributions in Belgium, Computers, Environment and Urban Systems, 31, 2007, 188-205.

FLAMM, R. O., TURNER, M. G.: Alternative model formulations for a stochastic simulation of landscape change. En De Aranzábal, I., Schmitz, M. F., Aguilera, P., Pineda, F. D.: Modelling of landscape changes derived from the dynamics of socio-ecological systems. A case of study in a semiarid mediterranean landscape, Ecological Indicators, 8, 2008, 672-685.

IRWIN, E. G., GEOGHEGAN, J. (2001): Theory, data, methods: developing spatially explicit economic models of land use change, Agriculture, ecosystems and environment, 85, 7-23. 
LACITIGNOLA, D., PETROSILLO, I., ZURLINI, G.: Modelling socio-ecological tourism-based systems for sustainability. En De Aranzábal, I., Schmitz, M. F., Aguilera, P., Pineda, F. D.: Modelling of landscape changes derived from the dynamics of socio-ecological systems. A case of study in a semiarid mediterranean landscape, Ecological Indicators, 8, 2008, 672-685.

LE SAGE, J. P., KELLEY, R. (2009): Introduction to spatial econometrics, CRC Press Taylor \& Francis Group, Boca Raton, London, New York.

LOPEZ, L., LOIS, R., GUICHARD, F. (coord.) (1997): La articulación territorial de la Raya Hispano-portuguesa. Actas Simposium Vilar Formoso, Fundación Rei Afonso Henriques, Zamora.

LORENZONI, I., JORDAN, A., HULME, M., TURNER, R. K., O'RIORDAN, T.: A co-evolutionary approach to climate change impact assessment: Part I integrating socio-economic and climate change scenarios. En De Aranzábal, I., Schmitz, M. F., Aguilera, P., Pineda, F. D.: Modelling of landscape changes derived from the dynamics of socio-ecological systems. A case of study in a semiarid mediterranean landscape, Ecological Indicators, 8, 2008, 672-685.

MATTHEWS, R.: The people and landscape model (PALM): Towards full integration of human decision-making and biophysical simulation models. En De Aranzábal, I., Schmitz, M. F., Aguilera, P., Pineda, F. D.: Modelling of landscape changes derived from the dynamics of socio-ecological systems. A case of study in a semiarid mediterranean landscape, Ecological Indicators, 8, 2008, 672-685.

MORA, J. (coord.) (1997): Los ríos internacionales Tajo y Guadiana en el desarrollo integral de Extremadura, Alentejo y Beira Interior, Gabinete de Iniciativas Transfronterizas, Junta de Extremadura, Centro Europeo de Desarrollo Regional, Cáceres.

ROCHA, E. J.: A contribuição da cooperação transfronteiriça para o reforço da articulação territorial da Raia Ibérica. Comunicación en XI Coloquio Ibérico de Geografía, (Alcalá de Henares, 1-4 de octubre de 2008).

SERRA, P., PONS, X., SAURÍ, D.: Land-cover and land-use change in a mediterranean landscape: A spatial analysis of driving forces integrating biophysical and human factors, Applied Geography, 28, 2008, 189-209.

WANG, Y., ZHANG, X. (2001): A dynamic modeling approach to simulating socioeconomic effects on landscape changes. En De Aranzábal, I., Schmitz, M. F., Aguilera, P., Pineda, F. D.: Modelling of landscape changes derived from the dynamics of socio-ecological systems. A case of study in a semiarid mediterranean landscape, Ecological Indicators, 8, 2008, 672-685.

WU, J. AND HOBBS, R.: Key issues and research priorities in landscape ecology: an idiosyncratic synthesis. En De Aranzábal, I., Schmitz, M. F., Aguilera, P., Pineda, F. D.: Modelling of landscape changes derived from the dynamics of socio-ecological systems. A case of study in a semiarid mediterranean landscape, Ecological Indicators, 8, 2008, 672-685. 